\title{
Les Lumières de la transe. Approche historique du tarentisme
}

Gino L. Di Mitri

\section{(2) OpenEdition}

\section{Journals}

Édition électronique

URL : http://journals.openedition.org/ethnomusicologie/102

ISSN : 2235-7688

\section{Éditeur}

ADEM - Ateliers d'ethnomusicologie

Édition imprimée

Date de publication : 1 novembre 2006

Pagination : 117-137

ISSN : 1662-372X

\section{Référence électronique}

Gino L. Di Mitri, «Les Lumières de la transe. Approche historique du tarentisme », Cahiers

d'ethnomusicologie [En ligne], 19 | 2006, mis en ligne le 15 janvier 2012, consulté le 20 avril 2019. URL:

http://journals.openedition.org/ethnomusicologie/102

Ce document a été généré automatiquement le 20 avril 2019.

Tous droits réservés 


\title{
Les Lumières de la transe. Approche historique du tarentisme
}

\author{
Gino L. Di Mitri
}

1 Je me suis souvent demandé, dans l'hypothèse d'une histoire programmée de la transe et des états de conscience modifiée en Europe et en Méditerranée, si celle-ci était possible en circonscrivant chaque événement géoculturel en un chapitre spécifique. La réponse a évidemment été négative, et ceci pas seulement en raison de scrupules philosophiques. Ce constat dérive des affinités structurelles étroites - même si elles ne sont pas toujours symboliques - entre les phénomènes de possession ritualisée des trois continents bordant la Mare nostrum; que ces affinités procèdent d'une lointaine origine commune ou de relations et d'influences réciproques. Parmi les nombreux rituels de possession répandus un temps dans ce territoire bigarré, le tarentisme, hormis le fait qu'il est le plus connu de ces phénomènes en Europe, est aussi celui qui a duré le plus longtemps. Il est le seul à avoir survécu jusqu'à il y a une quarantaine d'années dans des régions d'Italie méridionale comme la Campanie, la Calabre, mais surtout les Pouilles et la Terre d'Otranto.

2 Expliquons d'abord ce qu'est le tarentisme: en quoi consistait ce rituel, comment il se déroulait et quels étaient ses acteurs. Nous partons de la considération préliminaire qu'il n'a jamais été classifié - sinon depuis Gilbert Rouget - comme un véritable phénomène de possession (Rouget 1990). Ernesto de Martino lui même, dans son célèbre livre La terra del rimorso (1961) ne l'a pas considéré comme tel, en en parlant comme d'un phénomène religieux « veiné de syncrétismes » : mais plutôt comme d'un fatras symbolique complexe refaçonné au cours des siècles à la lumière de la spiritualité chrétienne et de la pensée médicale européenne. De Martino voyait la transe comme une éventualité émotive induite par le paroxysme de la musique et de la danse, et non pas comme un véritable état de conscience modifié. A cet égard, l'ethnomusicologue Diego Carpitella est plus proche de la compréhension de l'essence du phénomène, comme en témoigne son importante étude publiée dans l'ouvrage de de Martino, où il parle du tarentisme comme d'un « exorcisme choréo-musical» (Carpitella 1961 : 335-372). 
3 Cela dit, rappelons que, selon les premiers témoignages médiévaux, le tarentisme était considéré comme une maladie produite par la morsure d'une araignée, la tarentule des Pouilles ou Lycosa tarentula, qui, en injectant son venin, provoquait un état de souffrance psychophysique croissante : rien d'autre, en somme, qu'une maladie bizarre caractérisée par des manifestations plutôt extravagantes chez les personnes qui en étaient frappées.

4 Le sujet affecté par ce syndrome présentait, en effet, un cadre symptomatique typique: regard fixe et ahuri, migraines, nausées, douleurs articulaires dans tout le corps et non seulement sur la partie touchée par la morsure, vomissements, états léthargiques suivant l'agitation et la frénésie, parfois pyrexie, cachexie, bouche sèche, rétention urinaire ; sans oublier le priapisme chez les hommes et le délire érotisant chez les femmes. Mais ce qui surprenait les médecins, les savants et les observateurs était le fait que l'expression ritualisée de la souffrance de chaque homme ou femme affecté de tarentisme avait des caractères individuels, spécifiques, uniques et, de toute façon, extrêmement variables. Les deux symptômes constants du tarentisme étaient donc l'hébétude suivant la morsure et la varietas comportementale ${ }^{1}$. La chose était bien connue de Léonard de Vinci qui, dans son Bestiaire, avait consacré au tarentisme son aphorisme: "La morsure de la tarentule maintient l'homme dans sa résolution, c'est-à-dire dans ce qu'il pensait quand il a été mordu» (Marinoni 1952)2. La morsure bloque donc le sujet dans sa pensée et son comportement en le liant - dit de Martino - «à l'épisode mythique non résolu ». Comme l'a justement écrit Gabriele Mina, si le diagnostic de Léonard de Vinci est d'ordre symbolique, l'interprétation de ce phénomène par de Martino est de nature psychologique et allégorique, comme si le blocage était une sorte de réponse symbolique à la crise de remords (Mina 2000) ${ }^{3}$.

5 Mais, présentée en ces termes, l'explication est insuffisante: il y a autre chose qui apparaît sur le fond de ce décor, entre les hurlements et les danses, sous les coups pressants des tambourins et dans le son hypnotique des violons, quelque chose qui rappelle le théâtre inquiétant de la possession.

$6 \mathrm{Si}$, donc, à l'origine de la première crise de tarentisme, il y a un épisode traumatique, un conflit intérieur ou familier, un amour contrarié, un événement ayant déterminé une forme de culpabilité, ou si le malaise psychophysique suit un deuil, un abandon de la part du conjoint, une douleur inconsolable, alors la morsure - avérée ou présumée - d’une araignée, d'un reptile, d'un scorpion, ou la simple vue de ces animaux symboliques de l'univers culturel primitif, provoque la chute dans l'hébétude et l'explosion de la varietas comportementale.

7 Il y n'a aucun doute que ces deux éléments - le regard hébété d'une personne en crise et l'interprétation disparate et subjective du vécu à travers des convulsions encadrées et ordonnées en une chorégraphie progressivement dialoguée avec les airs joués par des musiciens - il y n'a aucun doute que ces deux éléments appartiennent à la possession. Nous les retrouvons dans la derdeba maghrébine, dans le zar éthiopien ou dans le ndoep sénégalais. Et, pour renforcer cette affinité, intervient aussi l'élément du chromatisme : comme les saints du maraboutisme populaire musulman ont leurs couleurs électives spécifiques; de même, les tarentules des Pouilles avaient des couleurs sur la base desquelles il était possible de diagnostiquer la typologie de la souffrance, d'indiquer aux musiciens la musique et le rythme les mieux adaptés à la thérapie et de reconnaître probablement l'esprit caché derrière cette tarentule. On sait comment le corybantisme grec connaissait aussi ce chromatisme, comme le relève Gilbert Rouget ; mais ce n'est pas 
le cas de nous arrêter, ni de réitérer la dérivation du tarentisme de cet aspect de la spiritualité de la Grèce antique.

8 Ce qui est plus intéressant est de retrouver les signes de la possession dans la littérature scientifique allant de la fin du XVII siècle à la fin du XVIII siècle, et de mettre en évidence comment, durant cette période, les observateurs les plus attentifs et les analystes européens du tarentisme eurent sous les yeux un rituel dont la forme n'était pas encore dégradée ni privée de sa richesse symbolique, pas encore réduite à cette épave que de Martino enregistra sur le terrain pendant son enquête à Galatina en 1959.

La première source documentée qui dévoile le caractère de possession du tarentisme nous vient de Tommaso Cornelio. En 1670 cet académicien napolitain réalisa un voyage dans les Pouilles dont il tira des observations à propos du tarentisme qu'il envoya deux ans plus tard aux Philosophical Transactions. Il y a dans cette lettre d'importantes informations permettant d'établir le rapport - jamais suffisamment approfondi - entre empoisonnement réel et possession, entre mélancolie pathologique et dissimulation, entre attitude rationaliste des savants et crédulité. Ce document nécessite une relecture et un décodage pour être appliqué aux sujets ici traités. Voici la première partie du récit de Tommaso Cornelio ; elle a pour protagoniste un sujet mordu par une araignée et mort après d'atroces souffrances.

[...] en Terre d'Otrante, où ces insectes très sont nombreux, il y avait un homme qui, croyant avoir été mordu par une tarentule, montra sur son cou une petite tache, autour de laquelle se formèrent en peu de temps des pustules pleines d'un liquide séreux. Quelques heures plus tard, ce pauvre homme fut grandement tourmenté par de violents symptômes tels que syncopes, grande agitation, vertiges et vomissements ; pourtant, sans quelque tendance à danser ni aucun désir d'avoir des instruments de musique, ce malheureux mourut au bout de deux jours (Cornelio 1672 : 4006-4007).

Vraisemblablement, l'homme a été mordu par une araignée appartenant à une espèce dangereuse. Les manifestations ayant suivi la lésion nous font pencher pour un Latrodectus tredecimguttatus ou, plus probablement, pour un des autres arachnides au venin mortel encore aujourd'hui répandus dans la péninsule Salentine: le Loxosceles rufescens (Dufour 1820), le Cheiracanthium punctorium (Villers 1789) ou le Cheiracanthium mildei (Koch 1864). En excluant le premier, dont les symptômes de la morsure se réduisent à une activité vénéneuse du type nécrotico-émolitique concentrée dans la zone intéressée, et qui n'évolue que rarement vers la forme systémique appelée viscérocutanée, par de nombreux côtés semblable au syndrome "porpora trombotica trombocitopenica de Moschowitz ", les soupçons se tournent vers les deuxième et troisième, qui appartiennent à la famille des Clubonidae, ne serait-ce que par la coïncidence de la symptomatologie (malaise et vomissement) et surtout par l'entité de la lésion cutanée. Il est donc certain que le cas clinique reporté par Tommaso Cornelio ne fut pas un arachnidisme de Lycosa tarentula, mais quelque chose de beaucoup plus grave (Pepe 2002).

11 L'importance de la lettre de Tommaso Cornelio aux Philosophical Transactions réside cependant dans le lien étroit entre les comportements d'arachnidisme et les comportements de possession, un lien qui n'a jamais été considéré d'une manière adéquate par les spécialistes. En effet, après avoir raconté l'agonie et la mort de l'homme d'Otranto, l'auteur, qui ne savait évidemment pas qu'il se trouvait en présence d'une véritable morsure de Latrodectus, de Cheiracanthium ou de Loxosceles, écrivait : 
Cette même personne m'a déclaré que tous ceux qui pensent avoir été mordus par des tarentules (sauf ceux qui feignent de l'être pour quelque raison) sont pour la plus grande partie des jeunes femmes faciles [que l'auteur italien appelle «Douceurs de sel»] qui, en tombant dans cette folie mélancolique en raison de quelque indisposition spéciale, se convainquent selon le préjugé populaire d'avoir été piquées par une tarentule. Et je me rappelle avoir observé en Calabre que certaines femmes, frappées par un accident comme celui-ci, étaient considérées comme possédées par le démon ; et il est commun dans cette province de croire que la plus grande partie des maladies qui tourmentent le genre humain dérive des mauvais esprits (Cornelio $1672:$ 4006). - comme toute autre maladie - est attribué à la possession d'un esprit, d'un animalsymbole, d'un démon ou - ajoutons-nous - d'un saint; d'une façon semblable à ce qui a été observé jusque bien au-delà de la première moitié du XXe siècle dans des zones de la Méditerranée chrétienne comme Galatina, où le binôme saint Paul/tarantule, abondamment traité par Ernesto de Martino, et celui de saint Paul/serpent, récemment étudié par d'autres auteurs (Montinaro 1996), s'alignent parfaitement sur la dynamique des rituels de possession et de guérison observés dans le monde islamique, asiatique et africain (Rouget 1990; Lapassade 1976; de Heusch 1971). Dans les publications de vulgarisation du XVIII ${ }^{e}$ siècle, chaque référence faite par un médecin aux états de possession par le démon ou des esprits malins apparaissait comme un élément à traiter avec extrême prudence. Ainsi s'explique le fait qu'en 1760, presque un siècle après sa sortie dans l'organe officiel de la Royal Society, la lettre de Tommaso Cornélius était reprise par le Journal Économique de Paris, mais censurée des lignes attribuant chacune des maladie affectant ces femmes pauvres et «ignorantes » du midi de l'Italie aux entités démoniaques; que ceci fût par respect pour le rationalisme dominant ou pour raisons d'opportunité par rapport aux lois de l'Église. 
Fig. 1 : La terre du tarentisme selon Kaspar Schott, Magia universalis naturae et artis (Bambergae 1659).

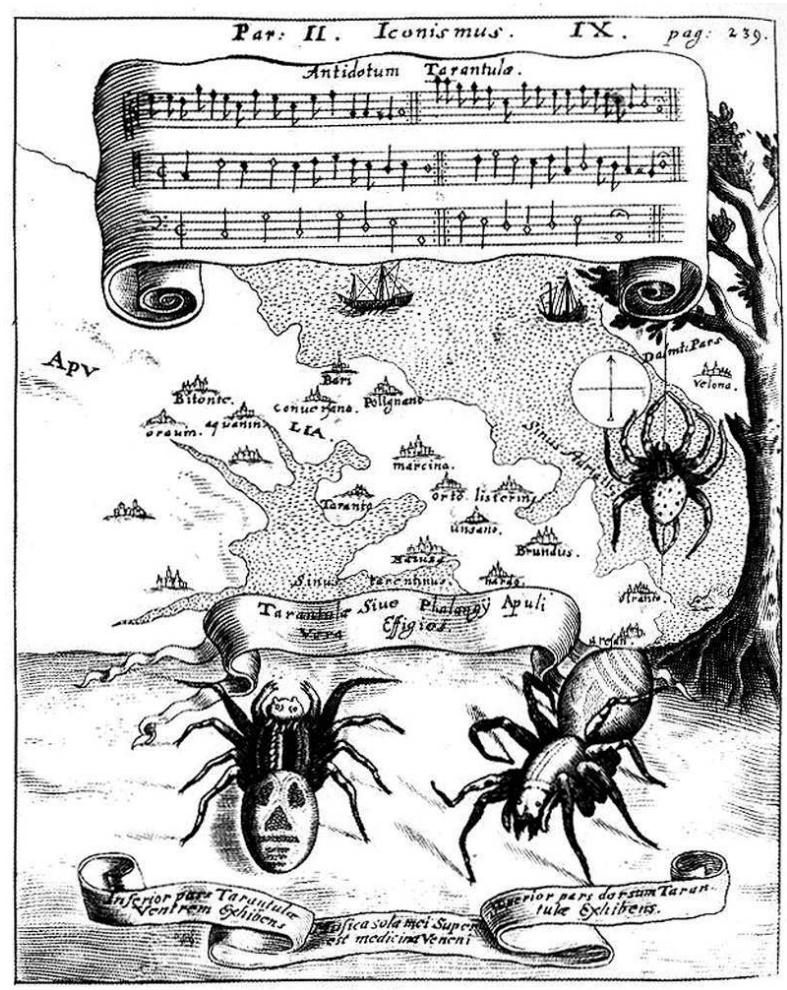


Fig. 2 : La Nosologia methodica de François Boissier de Sauvages de la Croix, le premier ouvrage qui essaya une classification médicale des différentes formes de tarentisme selon les principes de Carl von Linné.

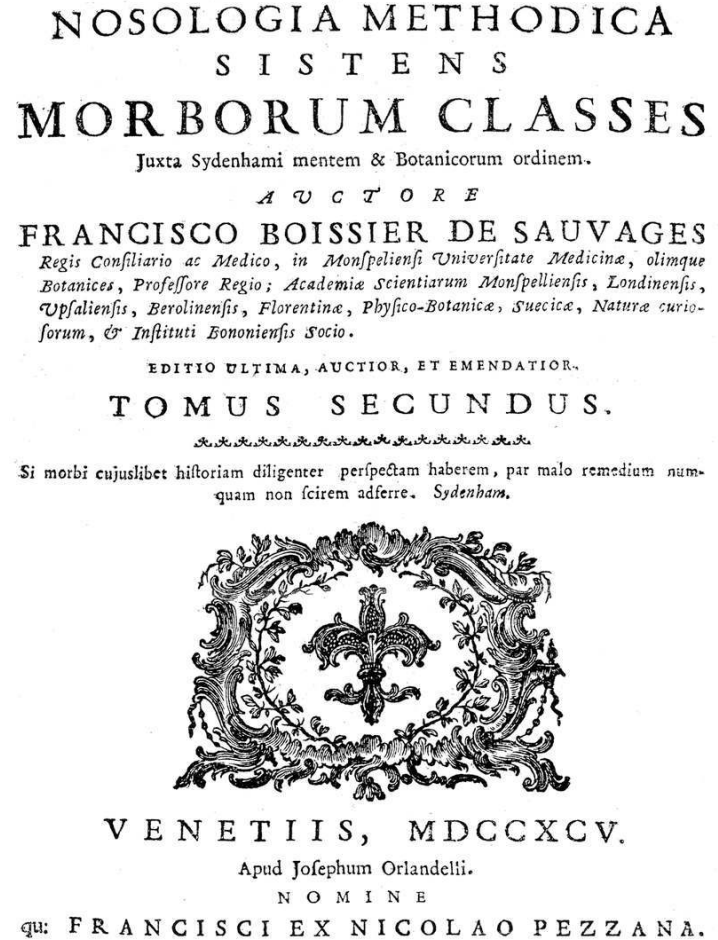

VENETIIS, MDCCXCV. phalangio apulo, un traité de 1706 du moine célestin Ludovico Lavalletta. Dans sa réponse à une objection avancée par des adversaires sceptiques, qui soupçonnaient que le tarentisme fût un prétexte pour danser et développer des rituels païens, le religieux écrivait :

Il est vrai, cependant, que les habitants des Pouilles se consacrent peut-être plus profusément et plus immodérément à la danse que les autres peuples, surtout en temps d'été et qu'ils courent en cercle en exultant gaiement dans les chapelles, aux carrefours et dans les cours; mais dans ce cas ils ne sont pas poussés par cette étroitesse intérieure de l'âme, par la compression et par tous les autres symptômes par lesquels sont tourmentés ceux qui sont attaqués par les tarentules (Valletta $1706: 110-112)$.

13 La réponse de Valletta, par les différents éléments qu'elle contient, est très importante du point de vue anthropologique: a) elle introduit clairement l'idée de ritualisation d'un phénomène ; b) elle établit une comparaison avec la prisca religio ${ }^{4}$ des anciens; c) elle confirme, en utilisant des observations ethnographiques, le diagnostic sur le tempérament des habitants des Pouilles énoncé quelques années auparavant par Giorgio Baglivi ; d) elle atteste l'existence d'une dévotion populaire faite aussi de danses autour des petites églises, de fêtes patronales ou de célébrations en l'honneur de saints tutélaires; e) elle détache radicalement la danse thérapeutique de celle de dévotion, réaffirmant la véracité de l'effet vénéneux de la tarentule.

14 Mais c'est en décrivant analytiquement la danse de la tarentule que Valletta relève peut-être involontairement - un détail qui place le tarentisme dans le registre de la possession. Dans la littérature biomédicale d'influence cartésienne des XVII et XVIII ${ }^{\mathrm{e}}$ 
siècles, le tarentisme se présente comme l'exemple parfait d'une cause naturelle agissant sur l'intelligence et produisant ces perturbations. Dans le sillage de cette physiologie, Ludovico Valletta a le mérite de consigner quelques-unes des premières observations d'états modifiés de conscience chez des patients atteints de tarentisme et suivis directement dans leur crise.

Si je peux raconter quelque chose de l'agitation extraordinaire et intense qui prend tout le corps, j'ai moi-même vu une femme tellement tourmentée par le venin qu'elle était prise par une fièvre violente, dominée par des fantômes furibonds, ou mieux, possédée par des démons arrogants accourus l'envahir, en entendant le son des instruments de musique, elle se précipitait en sauts d'excitation ; et ainsi, dans la dense agitation, de tous les membres du corps c'était surtout la tête qu'elle secouait et qu'elle faisait tournoyer d'un côté à l'autre ; tant et si bien que mes yeux et ma tête, contaminés par la même agitation, souffraient de vertiges (Valletta 1706 : 74-75).

On voit transparaître dans ce passage la qualité d'exorciste du moine Valletta. Ses références à la possession révèlent un arrière-plan de connaissance des possédés, et nous sont utiles pour associer le comportement de la femme aux techniques de la transe. En effet, les gestes qui consistent à secouer et à faire tournoyer la tête nous apparaissent comme un dispositif mis en marche intentionnellement par l'acteur du rituel de possession pour favoriser l'apparition d'états d'altération de conscience. Gilbert Rouget fait remonter au Timée de Platon les deux grands principes de la thérapie choreutique coribantique ${ }^{5}$ : le balancement rythmique et l'antagonisme entre les mouvements extérieurs et intérieurs. De ces mouvements il évoque aussi l'acte de danser en jetant brusquement la tête en arrière, comme dans les rituels dionysiaques ou dans certaines descriptions de la littérature mystique arabe, où l'état de transe est atteint grâce l'agitation de certaines parties du corps. L'explication des troubles psychiques soignés par ces rituels - que ce soit dans l'œuvre de Platon ou dans les sources orientales - ressemble beaucoup au processus par lequel l'âme cartésienne est effrayée et écrasée par les passions. Lus de l'extérieur selon des catégories anthropologico-médicales, ces mouvements d'auto-induction de la transe découlent de techniques corporelles; il s'agit néanmoins d'un dispositif visant la réalisation d'un état modifié de conscience obtenu par l'union de la musique et de la danse : une auto-excitation assez particulière, selon Rouget, car elle met en œuvre en même temps le souffle, une certaine sur-stimulation des cordes vocales, des mouvements très accentués de rotation de la tête et toute une gestuelle qui, sûrement, consomme (ou libère) beaucoup d'énergie.

Le tarentisme, outre le fait qu'il est un syndrome très complexe, est cependant aussi un système thérapeutique articulé. Valletta livre à la dimension diaphorétique ${ }^{6} \mathrm{du}$ corps engagé dans l'expulsion des humeurs toxiques à travers la sueur de l'agitation et de la danse, une série d'attitudes et de techniques qui concernent en réalité l'auto-induction de la transe. L'analyse de Gilbert Rouget est précise: la transe serait un changement d'identité et, dans son déroulement, elle apparaît comme le vécu d'une identité autre. La musique serait le seul langage capable de parler en même temps à l'esprit et au corps en leur permettant de s'exprimer sous forme de danse. Et le rythme, grâce à cette agitation et à la fréquence du recours à l'accelerando et au crescendo, aurait le pouvoir de créer un état d'effervescence particulièrement propice - les raisons en sont claires - à l'apparition de la transe. Valletta écrit :

« [Une] pauvre femme avait suspendu au plafond de sa maison une corde, dont elle serrait fortement le bout avec les mains ; elle s'était jetée sur la corde en l'agrippant complètement et, les pieds détachés de terre, elle faisait rapidement tourner sa tête 
de partout, avec une attitude furibonde, les cheveux ébouriffés, le visage enflammé et les yeux torves; j'étais stupéfait et ne comprenais pas qu'avec ces soubresauts si rapides et violents de la tête et le vertige qui en advint, elle ne tombât pas au sol » (Valletta $1706: 75)$.

L'analyse du récit de Valletta ne tient pas compte des aspects rituels et culturels de la thérapie du tarentisme; elle se concentre sur ses aspects purement médicaux, même si nous devons admettre que le dispositif d'auto-induction de la transe à travers la rotation et le secouement de la tête, le balancement sur la corde et même le remède, attesté par Athanasius Kircher, consistant à se faire bercer par la mer dans un bateau, ont été interprétés de façon erronée comme les moments d'une représentation à l'intérieur du rite. Ernesto de Martino a voulu faire remonter le balancement, sur la corde ou d'autres instruments de suspension motrice comme la balançoire ou le berceau, à un aiôresis symbolique des tarentulés 7 . Epifanio Ferdinando ("pensilem en cunam moveri cupiunt »), Giorgio Baglivi («motum pensilem amant») et Athanasius Kircher (tarentulés suspendus aux branches des arbres la tête en bas) seraient les exemples d'un symbolisme remontant à la spiritualité de la Grèce antique, mais dans lequel de Martino ne réussit pas à entrevoir les caractères uniques du dispositif auto-inductif de la transe. Le concept de transe est, au contraire, absent de la Terra del rimorso, tout au plus affleure-t-il occasionnellement des descriptions de l'expression faciale des tarentulés. Il est de même absent du traité du médecin Francesco De Raho, Il tarantolismo nella superstizione e nella scienza (1908) ${ }^{8}$; cette description est cependant longue, minutieuse et caractérisée par des analogies extraordinaires avec les passages de Ludovico Valletta sur le balancement.

Fig. 3 : Description comparative du rituel maghrébin des jnouns par Boissier de Sauvages de la Croix.

4. Tarantifmus Tingitanus; le Janon.

St. Gervais in libro cui titulus eft Mèmoires hifforiques, refert aput Africanos, Tuneti fcilicet, endemim efle Tarantifmum fpontaneuni, feu a nuliws infeEti morfu dependentem, mulieribus potiflimum familiaren, quo ad faltandum, \& choreas agendas impelluntur, \& huic morbo nomen elt le Janon; hos quidem motus tanquam convullivos Auctor reputat; verum verofimile eft, cos ejufdem generis, ac quos vere tarantari apud Apulos edere folent.

In oppido Donzere dikto apud Delphinates idem mox invaluit in curando anthrace, ac olim in oppido Roquecourbe juxta Caltres, fcilicet incilo tumore, \& appofito fale, aceto ac pipere, xger ad faltandum \& choreas ducendas per biduum, rintinnabulorum fono, amicorum exemplo incitatur.

Un naturaliste du XVIII e siècle, Antonio Minasi, faisait remonter sans hésitation les danses des tarentulés aux orgies de Bacchus et Cybèle, et il cueillait dans le décor du rite 
les preuves de cette filiation. Selon lui, la maladie des habitants des Pouilles était volontaire. Comment expliquer autrement que Pline, Martial ou Horace n'en faisaient pas mention? Et, à propos des variables environnementales, il croyait que le climat et la géologie n'avaient pas changé. Et encore, en référence à plusieurs facteurs plus proprement ethnologiques, il affirmait que les danses populaires existaient aussi dans l'Antiquité. Finalement il était convaincu qu'alors, comme à son époque, les préjugés et l'imagination dominaient et que - comme en témoigne Théophraste - on croyait que la musique était un médicament puissant pour guérir de nombreux maux, y compris les morsures des bêtes venimeuses. Pourquoi donc, se demandait Minasi, le tarentisme n'avait-il pas éclaté aussi dans l'Antiquité classique? Les auteurs anciens, pensait-il, avaient souvent parlé de Tarente dans leurs œuvres et, si cette institution bizarre existait déjà, ils en auraient certainement fait mention. Cependant, comme le confirmait Minasi, il faut remonter à l'âge gréco-romain pour découvrir les vraies racines du phénomène. Et voici les indices clairs de cette origine lointaine :

1. les tarentulés ornaient l'endroit dans lequel se déroulait leur rituel avec des feuilles de vigne : Minasi ne se limite pas à reporter cette affinité claire avec le dionysisme déjà décrit par Baglivi, mais il précise qu'il a personnellement constaté cela «dans beaucoup d'observations faites sur des tarentulés »;

2. la préparation du décor comprenait aussi des rubans colorés, comme cela se faisait anciennement dans les cultes de Bacchus et de Cybèle, « la Terre sur laquelle on cultive aussi la vigne »;

3. les tarentulés s'habillaient de vêtements blancs ornés de rubans rouges, comme le faisaient déjà les bacchantes ;

4. le foulard blanc était un autre objet du rite, comme chez les prêtres de Cybèle, qui le portaient sur les épaules ou noué autour cou ;

5. un comportement courant parmi les tarentulés était le trachélisme, c'est-à-dire le secouement de la tête, de haut en bas et latéralement, comme le faisaient les bacchantes dans leur danse ;

6. les couleurs d'élection des tarentulés, spécialement des femmes, étaient - comme chez les adeptes des confréries dionysiaques - le rouge, le vert et le jaune : en particulier à Tarente, où l'usage était de porter des vêtements pourpres (en italien, tarantinidie)pendant les cérémonies orgiastiques ;

7. dans la danse des tarentulés, Minasi reconnaissait « les virevoltes, les gestes, les sauts, les battements de pieds sur le sol, les mouvements de la tête et toute l'artificieuse torsion des corps, avec soupirs répétés suivant les cadences du son, qui étaient déjà pratiqués en Lydie, en Phrygie, et par d'autres peuples d'Asie, dont ils reconnurent l'introduction, outre chez les Tarentins, chez les Grecs et les Romains » (Atenisio Carducci 1771 : 475-490) 9 .

Les sept éléments de comparaison diachronique entre tarentisme et dionysisme déterminés par Minasi méritent un bref commentaire. Il avait observé sur le terrain la thérapie musicale, presque sûrement à Tarente ou dans les régions limitrophes : s'il avait réussi à voir un périmètre rituel orné avec les objets et la végétation qu'Ernesto de Martino attribue au prétendu "tarentisme originel », c'est-à-dire à ce que Baglivi et Valletta avaient observé entre la fin du XVII ${ }^{e}$ et le début du XVIII ${ }^{e}$ siècle, cela signifie que la richesse et la complexité scénographique de ce dispositif thérapeutique étaient encore utilisées dans les années 1770, et elles doivent avoir peut-être survécu, du moins dans les campagnes des Pouilles, jusqu'aux premières décennies du XIX ${ }^{\mathrm{e}}$ siècle. Parmi ces objets décoratifs, il semble déduire que les rubans colorés ne sont pas seulement des instruments de diagnostic de la maladie (rudimentaires si on les compare avec la 
médecine officielle, raffinés si on les circonscrit à l'univers magico-populaire dans lequel ils servent de réactifs placés à côté de l'« épreuve » d'exploration des modes musicaux) ; mais qu'ils se réfèrent à un horizon spirituel lointain : le culte de Dionysos. S'il en est ainsi, sarments de vigne et rubans polychromes sont alors des exemples remarquables de survivance de longue durée.

20 L'habit des fidèles de saint Paul et de la tarentule est blanc: cette information, donnée par Minasi, est très importante car on avait jusqu'alors considéré que l'habit blanc était lié à une tradition purement dévotionnelle chrétienne. Vittorio Macchioro a insisté sur ce détail avec des arguments convaincants depuis les années 1920-1930: le blanc était la couleur choisie et endossée par les adeptes de la religion orphique durant les commémorations de la passion de Dionysos. Macchioro traita ponctuellement cet aspect dans Orfismo e Paolinismo (1924: 259-260), mais il établit dans Zagreus (1930: 208) une analogie plus étroite entre les cultes orgiastiques et extatiques et le tarentisme. Son gendre Ernesto de Martino saura plus tard valoriser cette information, sans pourtant approfondir une problématique restée - avant et après la Terra del rimorso - au vague stade d'inventaire des antécédents.

21 Venons-en au trachélisme, qui est peut-être l'aspect le plus important signalé par Minasi. On se rappellera que, pour le moine exorciste Ludovico Valletta, le secouement de la tête de la part des tarentulés était considéré comme un comportement horripilant causé par la virulence du venin. Antonio Minasi estime par contre qu'il s'agit plutôt d'une coquetterie féminine, celle de tenir les "cheveux déliés au vent»: un caprice extravagant, connu et pratiqué par les bacchantes. Mais ce geste s'inscrit aussi dans la longue durée, non pas pour des raisons étroitement chorégraphiques, mais par le fait qu'il semble être un extraordinaire coefficient inductif de la transe, adopté de tout temps dans les danses de possession de nombreuses civilisations. Du point de vue physiologique, cet effet a efficacement été décrit par Andras Zempléni (le travail de la crise consiste - cet avis est partagé par Gilbert Rouget dans ses recherches sur le terrain - en une séquence motrice qui a relevé des exemples de cette pratique engageant initialement tout le corps du danseur en un crescendo; ce mouvement paroxystique se concentre ensuite dans la tête, qui est soumise à une oscillation violente du haut vers le bas ou de droite à gauche) (Zempleni 1966 : 295-439).

Cette phase est souvent accompagnée, et presque toujours suivie, de gestes désordonnés, de contorsions et de cris, puis de la chute typique de la transe de possession. La cause physiologique de cette chute est l'autostimulation vestibulaire qui, unie à l'accroissement du volume sonore et à l'accélération du rythme, porte à l'épuisement musculaire (lequel dérive peut-être de la danse agitée) et à la perte d'équilibre. Le vestibule du labyrinthe est, en effet, cette partie de l'oreille intérieure située dans la cavité centrale de l'os temporal qui connecte le labyrinthe osseux avec les canaux semi-circulaires. La sollicitation motrice de l'utricule et du saccule, qui dépendent du labyrinthe membraneux, a des répercussions évidentes sur le sens de l'équilibre : voilà comment, aujourd'hui, on s'explique la chute des tarentulés au cours de la danse, et surtout comment la musique, le rythme et les représentations subjectives interagissent dans ce processus d'altération des états ordinaires de conscience. Il faut cependant insister sur le fait qu'aucune transe ne peut advenir si ce processus n'est pas inscrit dans un contexte rituel. Entre la physiologie de l'oreille exprimée par le médicament iatromécanique, selon laquelle la porte d'entrée des effets thérapeutiques était l'organe auditif, mais dans la mesure où les sons se propageaient des vésicules du labyrinthe aux tubules des nerfs et, 
de là, aux humeurs et aux parties solides du corps, et la physiologie des passions de Francesco Serao, il y a un hiatus que l'égalisation occasionnelle de Minasi entre le trachélisme des bacchantes et le mouvement de la tête des tarentulés n'aurait de toute façon pas réussi à combler. L'état spécial d'ébriété dérivant de l'excitation motrice du labyrinthe aurait été notée par Henri Aubin et, après lui, empruntée par Michel Leiris. Mais le père Antonio Minasi a peut-être été le premier à avoir une intuition qu'il aurait dévoilée, deux siècles plus tard, sur la physiologie de la transe.

Fig. 4 : Ménade dansant tirée d'une peinture vasculaire apulienne du Ve siècle avant J.-C. (Karlsruhe, Badisches Landesmuseum).

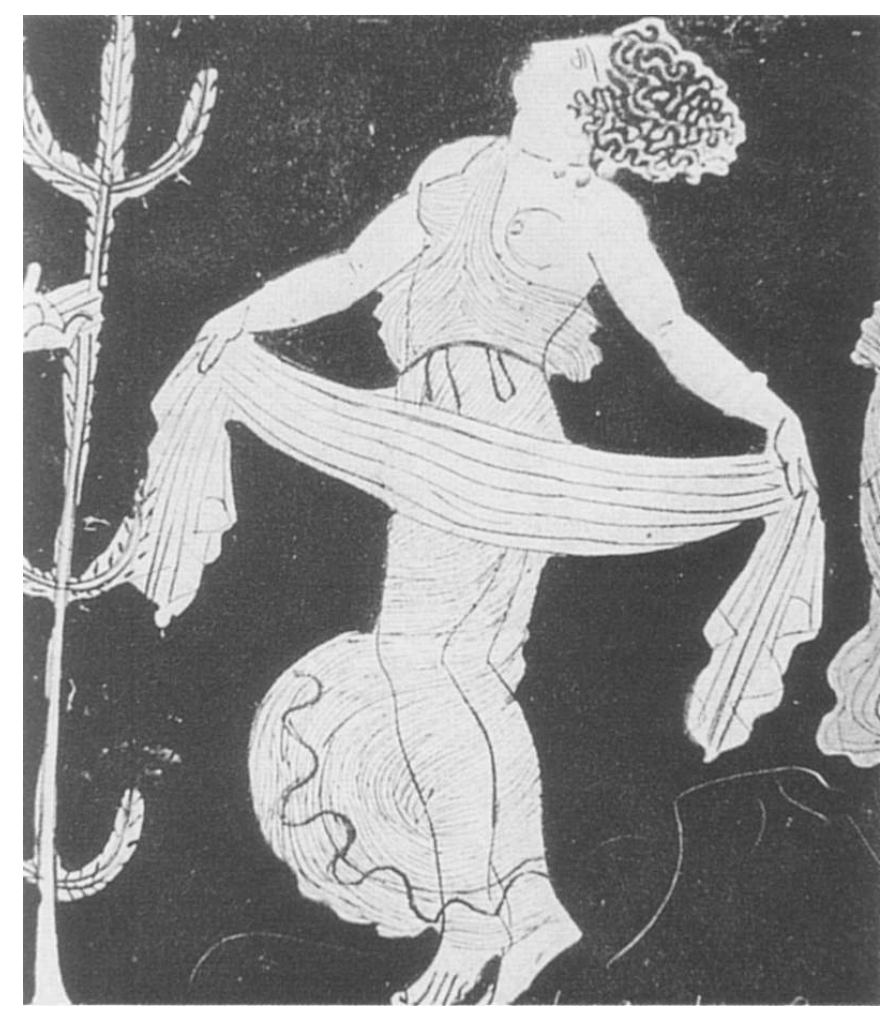


Fig. 5 (p. 129) : Rodolfo Muller (1802-1885), Tarantella. Galatina, collection Mario Congedo.

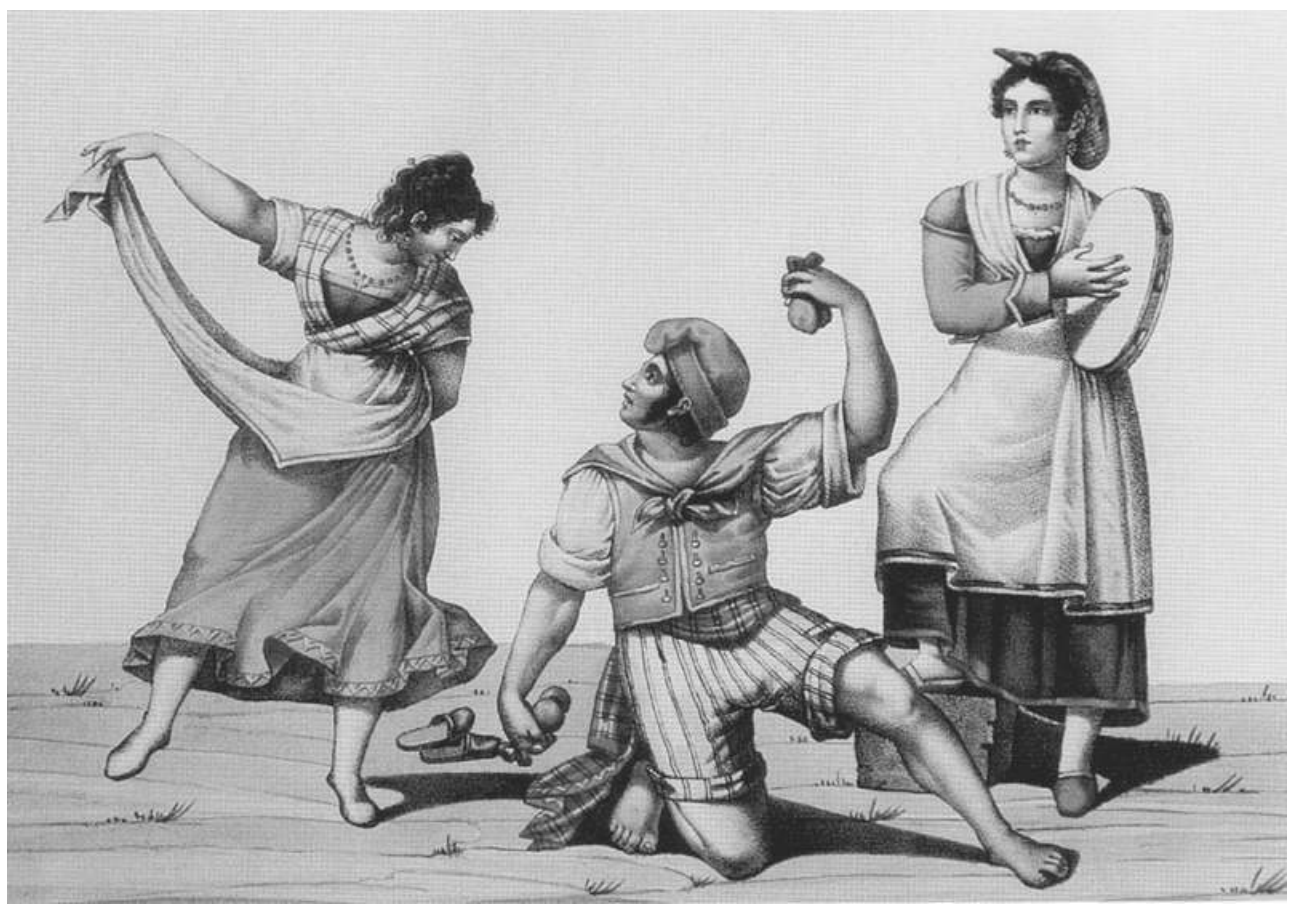

En un rapide survol du problème des couleurs électives, domaine des représentations symboliques, faisons une dernière référence à la description de la danse. Il n'est pas clair si l'artificieux tourbillon du corps signifie pour Minasi un exhibitionnisme simple des danseurs ou un mouvement tendant à produire ces états altérés de conscience que Harald

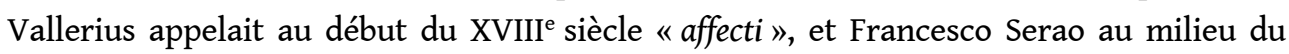
même siècle "passioni »: il est un fait que celui-ci et d'autres comportements semblent descendre de coutumes des anciens peuples euro-asiatiques transmis aux Grecs et aux Romains, et de ceux-ci aux Tarentins. On sait comment la contagion chorétique se transmit par des Tarentins prisonniers, déportés dans la capitale par les Romains après la guerre victorieuse contre Pyrrhus et objets d'un célèbre senatus consultum sur les bacchanales.

Le fait qu'Antonio Minasi vitt l'origine du tarentisme dans les cultes de Dionysos et de Cybèle n'était pas une conjecture arbitraire ni le fruit d'une séduction irrésistible du climat culturel néoclassique qui l'environnait. Dans sa tentative de comparaison, Minasi se fiait au Lexicon de Suida, aux commentaires de Johann Frederik Reitz à Lucien de Samosate, et surtout à la littérature sur les fouilles d'Herculanum qui, depuis la fin des années $40 \mathrm{du} \mathrm{XVIII}{ }^{e}$ siècle, étaient de plus en plus intenses. Favorisé par la proximité des chantiers dont avaient émergé les fresques sur les mystères de la religion païenne, Minasi va en premier lieu chercher, parmi les ruines, à les comparer avec la gestuelle des tarentulés; puis il adopte les observations d'Ottavio Antonio Baiardi $(1752 ; 1754$ et 1757-1765) et, probablement aussi, les écrits de Marcello Venuti (1748 et 1749), de Giuseppe Maria Mecatti (1752), de Charles Nicolas Cochin (1756), de Scipione Maffei (1748), de Giuseppe Bartoli (1762) et d'Anton Francesco Gori (1756). C'est une expérience pionnière de confrontation iconologique, qui sera poursuivie par Macchioro sur la voie tracée par Aby Warburg, et dont profiteront certains neuropsychiatres des années 1960 (Mora 1963 : 417-439). 
Mais c'est avec la Nosologia methodica de François Boissier de Sauvages de la Croix que le tarentisme entre dans un schéma de classification bien défini. Sauvages, qui ne cachait pas une certaine sympathie pour l'animisme de Georg Ernst Stahl, insère ce syndrome parmi les formes de folie, et plus précisément dans la huitième classe nosologique : celle des soi-disant «vesaniae»; mais il la distingue des autres car elle se rapproche des " morositates ", c'est-à-dire de ces formes de récidivité cyclique attestées par l'électivité saisonnière des tarantulés. Ce qui est nouveau et important dans cette interprétation du tarentisme consiste en la substitution de l'idée vague stahlienne de « faute » à l'origine de la souffrance mentale par une autre qui met le conflit intérieur au premier plan. Au domaine stahlien de l'âme succède l'illuminisme de la raison; l'imperfection dérive de l'état inculte des facultés rationnelles, qui ne règlent plus l'accord entre les actions individuelles et la conscience, entre l'intelligence et l'instinct. En insérant le tarentisme parmi les troubles mentaux et en devinant les causes de sa nature intimement conflictuelle, Boissier de Sauvages se trouve à l'origine d'une vision herméneutique qui culminera avec la psychanalyse sur le terrain réalisée en 1959 au Galatina par Giovanni Jervis, ainsi qu'avec la théorie du mauvais passé d'Ernesto de Martino.

Le mot "Tarantismus» de la Nosologia s'impose avec les travaux de deux autorités d'importance: Giorgio Baglivi et Francesco Serao. Le premier avait accrédité l'étiologie toxicologique, la symptomatologie choréo-convulsive et la thérapie musicale, propagées par l'opinion populaire; le second les avait démolies. Une fois terminé le rapport sommaire de la casuistique clinique et des caractères généraux pathologiques, l'auteur rappelle - en accord avec Serao et Minasi - que le tarentisme n'est mentionné par aucune source avant le $\mathrm{XV}^{\mathrm{e}}$ siècle; que ces mêmes araignées accusées d'en être la cause existaient avant cette époque et existent encore aujourd'hui dans tout le bassin méditerranéen jusque dans les régions les plus torrides des Pouilles sans que le phénomène soit attesté, ni par des observations des anciens, ni par de celles des contemporains.

Sauvages passe donc au classement des différents types de tarentisme, dont voici une synthèse schématique :

1. le Tarantismus Apulus Baglivi, subdivisé en différents embranchements parmi lesquels la tendance à danser nu, le « carnevaletto » des femmes, etc ;

2. le Tarantismus enteneasmus ou Enthusiasmus Galeni, auquel appartiennent la Danse de Saint Vito, la Danse de Saint Valentin, la Chorea, les aegri enterastici d'Hérodote et, de manière générale, toutes les danses médiévales de possession «sub velo devotionis » effectuées à l'intérieur ou près des églises ;

3. le Tarantismus musomania, c'est-à-dire l'amour pathologique pour la musique défini comme un trouble certainement de nature mélancolique dont - fait de modernité absolue Sauvages parle presque comme d'une maladie sociale frappant toutes les couches de la population et toutes les générations. C'est une «insanité épidémique " qui rend ses contemporains fous de mélodrame comme elle faisait délirer les anciens à l'écoute des tragédies d'Euripide; elle s'insinue dans les fêtes religieuses comme le carnaval durant le Carême, comme les danses labyrinthiques de la Tarasque lors des célébrations de sainte Marthe en Provence; elle frappe les gentilshommes cultivés comme les mélomanes fiévreux qui, au début du XVIII siècle, peuplent les pages de l'Histoire et Mémoires de l'Académie Royale des Sciences avec les récits de Mandajor et Dodart. La « vesania musica » assume le profil d'une névrose de masse ante litteram qu'il évoque, en lui proposant un traitement moral par des dosages savants d'un antidote homéopathique. La même musique que François Leuret fera administrer en 1833 aux aliénés de la Salpêtrière par Franz Liszt ; 
4. enfin - et nous voilà arrivés à un point d'importance cruciale - le Tarantismus Tingitanus ou Janon, qui ferme la liste de Sauvages et nous porte dans le monde des exotismes rituels de possession émergeant de la littérature missionnaire et coloniale. Mais dans ce cas c'est un médecin, et non pas un voyageur, qui relie entre eux le tarentisme des Pouilles et un culte maghrébin des génies et, à leur tour, ces phénomènes aux syndromes convulsifs. Sauvages appelle janon un rituel d'exorcisme pratiqué par des confréries féminines à Tunis, Tanger et dans d'autres villes d'Afrique du Nord: il le définit comme une sorte de tarentisme spontané, endémique et indépendant de la morsure d'insecte.

Dans le livre intitulé Mémoires historiques, Saint-Gervais dit qu'en Afrique, à Tunis, il y a un tarentisme spontané, non provoqué par la morsure d'un insecte, surtout commun chez les femmes qui se sentent poussées à sauter et à danser, et qui s'appelle Janon. L'auteur définit ces danses comme des mouvements convulsifs; il est en effet vraisemblable qu'elles soient du même genre que celles effectuées dans les Pouilles par les tarentulés (Boissier de Sauvages de la Croix $1795: 122-123)^{10}$.

Jacques Boyer de Saint-Gervais était un diplomate Français qui, en 1736, avait publié son livre de Mémoires historiques sur le Royaume de Tunis. Après l'avoir lu, je considère cet ouvrage comme le plus riche en données ethnographiques sur la Tunisie qui ait jamais été écrit sous l'Ancien Régime : une œuvre qui devrait aujourd'hui être prise en considération par quiconque - anthropologue ou ethnomusicologue - voudrait approcher de l'étude de cette réalité méditerranéenne. Mais voyons ce que Saint-Gervais écrit :

Il règne parmi les femmes une maladie fort commune et singulière, dont plusieurs meurent. Les gens du Pays l'appellent le Janou [en vérité le mot est imprimé "Janon », mais sur l'exemplaire que j'ai consulté à la Bibliothèque Municipale de Versailles quelqu'un a corrigé à la plume "Janou»]. Elle fatigue le corps de la malade par des mouvements convulsifs, qui l'agitent avec violence; durant ces accès une femme bat du tambour, et aux sons lugubres qu'il rend, essentiels à cette scène triste et comique tout ensemble, la malade danse, tourne avec rapidité, se dépouille de tous ses habits, s'affoiblit jusqu'à perdre la respiration, et tombe par terre, d'où elle est portée dans son lit, et parfumée avec toutes sortes d'aromates extrêmement forts. Les femmes attribuent cette maladie à une possession du malin esprit, qui s'empare du corps, et qui n'en peut être chassé qu'au bruit du tambour, ou par des caractères magique[s], qu'on applique sur différentes parties du corps de la malade, à laquelle dans cet état on ne refuse rien de tout ce qu'elle demande (StGervais 1736 : 193-195).

Ce rituel - extraordinairement semblable au tarentisme des Pouilles - n'est autre que le culte des jnoun, une croyance d'origine préislamique en des génies tenus responsables par la population de causer différentes maladies à travers la possession. L'origine du mot, justement proposée par Rouget, vient très certainement du latin genius. Qui souffre de troubles les plus disparates, physiques ou psychiques induits par la possession d'un génie se remet à la cure chorétique administrée par des confréries féminines qui «chassent » les entités spirituelles du corps des possédés avec la musique et la poésie: un procédé démontrant un caractère typiquement incantatoire. Dans ce sens, les possédés maghrébins répondent aux mêmes modalités thérapeutiques que celles exposées par Diego Carpitella dans son Esorcismo coreutico-musicale (1961). L'insertion de la part de Sauvages de ce tarentisme spontané dans le classement des vesaniae démontre donc que les médecins européens, qui opéraient dans la phase aurorale de la psychiatrie, considéraient ces phénomènes (qui n'étaient pas encore reliés par eux à des rituels et à des cultes de possession) comme appartenant à un schéma diagnostique commun de type convulsif, dont les symptômes extravagants ressemblaient aux hallucinations et aux 
représentations les plus variées et subjectives de phantasmata. Et bien qu'ils réussissent très bien à en déterminer les antécédents lointains dans l'automutilation sanglante des " Galli et Marsi ", dans les cultes de Cybèle et de Dionysos et en d'autres mystères anciens, les savants du XVIII ${ }^{e}$ siècle n'admirent jamais ouvertement le caractère de possession, bien que leur formation classique leur offrît l'exemple platonique des teletai katartikai dans lesquels était soulignée l'analogie entre manies "telestica, mantica et poieutica" communes autant au tarentisme qu'aux jnoun. Par ailleurs, Gilbert Rouget semble pencher pour cette analogie apulo-tunisienne quand il considère que le culte thérapeutique des jnoun, comme de la tarentule, appartient au domaine de la religion populaire, et qu'on y rencontre les mêmes indices de pratique magiques que dans le tarentisme. Ces pratiques peuvent en fait être observées sur un territoire allant des rives européennes de la Méditerranée jusqu'au Golfe de Guinée, même si les différences entre les confréries Tidjâniyya tunisiennes et les petits orchestres thérapeutiques du tarentisme sont moindres que celles entre les premiers et leurs homologues marocains et sénégalais.

31 Que la musicothérapie transcende le tarentisme et concerne aussi des pratiques collatérales à la chirurgie, mais surtout qu'elle prenne la forme d'une cure collective rituelle, émerge chez Sauvages du rapport de la cure de l'anthrax en différentes régions françaises :

Dans la ville de Donzère, dans la Drôme, une semblable cure est effectuée actuellement dans le traitement de l'anthrax, un temps aussi utilisée à Roquecourbe, près de Castres de la façon suivante : incisé le bubon et appliqué sel, vinaigre et poivre, incitez le malade à sauter et à danser pendant deux jours au son des tambourins et avec l'exemple des gens chers (Boissier de Sauvages $1795: 123$ ).

La iatromusique française possède des caractères intermédiaires entre le tarentisme des Pouilles, où le patient est placé au centre d'un périmètre rituel où on le fait danser, et le dispositif thérapeutique de l'argia sarde, où le patient reste par contre détendu et immobile au centre d'une place analogue et les parents dansent en cercle autour à lui.

En conclusion, Sauvages a deux mérites dans l'histoire du débat scientifique sur le tarentisme : le premier est d'avoir essayé, avec son classement nosologique, de fixer de solides points d'ancrage pour des énoncés qui risquaient toujours d'être entraînés à la dérive par l'incertitude et l'arbitraire; le second est celui d'avoir montré - peut-être inconsciemment - les traces d'un rituel encore présent autour du tarentisme en tant que maladie et que thérapie et, spécialement avec l'exemple de la cure de l'anthrax en France, d'avoir dévoilé la concomitance de théories et de pratiques médicochirurgicales et magico-empiriques dans le traitement de la maladie à la périphérie des Lumières.

Avec son classement du tarentisme, Sauvages se place à l'origine du dialogue interdisciplinaire entre neurosciences et anthropologie, et en particulier bien avant l'assomption - aujourd'hui complètement légitimée de la part des neurosciences - d'une interprétation comparative entre la terminologie chamanique et les effets des endorphines dans les phénomènes de transe de possession. Les neurophysiologues ont toujours eu peu d'intérêt à conjuguer la physiologie cérébrale aux aspects anthropologico-culturels dans l'étude des formes de souffrance mentale mystique, en situation rituelle et, d'une façon générale, dans les syndromes centrés sur des systèmes de croyances qui sous-tendent des situations conjoncturelles d'altération de conscience (Prince 1982: 299-302). Au contraire, la stérilité de nombreuses enquêtes anthropologiques dans ce secteur a été très souvent causée par l'inaptitude à acquérir les connaissances de base des neurosciences (Allovio et Favole 2001 : 73). L'interdisciplinarité 
inaugurée par Ernesto de Martino et Giovanni Jervis avait été, à bien y regarder, déjà tracée XVIII ${ }^{\mathrm{e}}$ siècle par des médecins comme Sauvages, et elle caractérise aujourd'hui le débat intense en ethnopsychiatrie ouvert par les recherches du doyen Vittorio Lanternari et poursuivies par Stanislav Deprez, Gérard Edelman, Harvey Whitehouse et Dan Sperber. Ce dernier a introduit en particulier l'idée d'épidémiologie des représentations (sur la base des théories sur l'esprit humain de Noam Chomsky et de Jerry Fodor), dans laquelle nous pouvons relever de fortes analogies avec le tarentisme (Deprez 1999 ; Edelman 1992 ; Whitehouse 1997 ; Sperber 1996).

Cette première intuition provenant de médecins linnéens, soucieux de définir les désordres de la psyché dans le cadre d'un classement nosologique qui reportât aussi les casuistiques dans les communautés locales et dans les populations exotiques, est devenue aujourd'hui une nécessité incontournable: le dialogue entre anthropologie culturelle, histoire de la maladie et sciences neuropsychiatriques fait l'objet, du moins à partir du milieu des années 1990, de réflexions épistémologiques denses. L'assumé qui, selon cette direction, devrait accompagner l'étude des phénomènes de psychopathologie contextuelle consiste à dire que le cerveau humain est pétri par un processus sélectif qui permet la stabilisation de certaines connexions au détriment d'autres. Pour ce faire, l'influence du milieu, de l'expérience et de la culture serait déterminante. À son tour, l'idée de sélection tient compte des choix culturels de l'individu; mais ceux-ci sont nécessairement restreints par le fait de son appartenance sociale. L'analogie extraordinaire entre les représentations formulées par les patients atteints de tarentisme et l'émergence d'autres syndromes connexes dans différents rituels de possession afroméditerranéens peut donc être lue, grâce à ces contributions, à partir de la théorie d'un modelage réductible à ce qui pourrait être défini comme la notion de «cerveau plastique ».

Cette plasticité consiste, à bien y regarder, en «la disponibilité relative de l'organisation neurologique à dialoguer avec le milieu environnant ». Si, comme des expériences sur des musiciens et des non-musiciens l'ont démontré, l'exécution et l'écoute de passages mélodiques comportent des degrés et des types variables d'excitation ou de sensibilisation de zones cérébrales bien précises; que la déclamation hypnotique et itérative de certains mots comporte la réduction de certaines zones cérébrales au profit d'autres, avec un glissement des zones cognitives de l'hémisphère gauche aux zones sensitives de l'hémisphère droit; que, de façon prévisible, ces effets peuvent également se produire sous l'effet de rythmes; que, enfin, des conditions complémentaires comme l'hyperoxie $^{11}$ au cours des mouvements chorétiques et les dynamiques psychomotrices précédemment décrites sont réunies à l'intérieur d'un contexte rituel, alors il apparaît clairement que le tarentisme évoque la possibilité d'une reconstruction nosologique qui tienne compte de facteurs intérieurs, physiologiques, et d'autres extérieurs, indubitablement culturels. Cette résolution fut fixée pour la première fois, quoique de manière encore sommaire, par François Boissier de Sauvages; elle est aussi un exemple de modelage: celui formé par les idées scientifiques environnant ses théories et ses pratiques.

Il apparait évident que ce tableau présente de fortes ressemblances avec les problématiques déterminées par la comparaison entre cultures occidentales « avancées " et cultures africaines, asiatiques, amérindiennes et océaniques traditionnelles jusqu'audelà de la fin de l'époque coloniale. Si Sauvages ressentait le besoin de comparer le rituel des jnoun au tarentisme, cela signifie qu'il considérait ces deux phénomènes comme 
apparentés, en dépit de leur éloignement culturel et géographique, et qu'il en reconnaissait le caractère psychopathologique par leur partage des caractéristiques de la mélancolie, de l'hypocondrie et de l'hystérie. Cela lui suffit pour déterminer, dans cet atlas encyclopédique de médecine que fut son Nosologia methodica, les traits distinctifs d'une tendance au comparatisme, qui donnera ses fruits de nombreuses décennies plus tard.

\section{BIBLIOGRAPHIE}

ALLOVIO Stefano et Adriano FAVOLE, 2001, «Attraverso le frontiere : uno sguardo antropologico alle neuroscienze », in Passaggi. Rivista Italiana di Scienze Transculturali : 73-85.

ATENISIO CARDUCCI Cataldantonio, 1771, Delle delizie tarantine libri IV. Opera postuma di Tommaso Niccolò d'Aquino Patrizio della Città di Taranto.Napoli : Stamperia Raimondiana.

BAIARDI Ottavio Antonio, 1752, Prodromo delle antichità d'Ercolano alla maestà del re delle Due Sicilie Carlo infante di Spagna, t. III. Napoli : Stamperia Palatina.

BAIARDI Ottavio Antonio, 1754, Catalogo degli antichi monumenti dissotterrati dalla discoperta città di Ercolano. Napoli : Regia Stamperia.

BAIARDI Ottavio Antonio, 1757-1765, Le pitture antiche d'Ercolano e contorni incise con qualche spiegazione. Napoli : Stamperia Reale.

BARTOLI Giuseppe, 1762, Lettera seconda [...] nella quale si pubblicano $i$ veri caratteri del noto busto, e si spiega una antica pittura d'Ercolano. Torino : F.A. Mairesse.

BOCCADORO Brenno, 2002, Ethos e varietas. Trasformazione qualitativa e metabole nella teoria armonica dell'antichità greca. Firenze : Leo S. Olschki Editore.

BOISSIER DE SAUVAGES DE LA CROIX François, 1795, Nosologia methodica sistens morborum classes juxta Sydenhami mentem et Botanicorum ordinem, t. II. Venezia : G. Orlandelli.

BOYER DE SAINT-GERVAIS Jacques, 1736, Mémoires historiques qui concernent le gouvernement de l'ancien et du nouveau royaume de Tunis [...] par M. de Saint-Gervais. Paris : Ganeau et Fils.

CARPITELLA Diego, 1961, "L'esorcismo coreutico-musicale del tarantismo », in de Martino Ernesto, La terra del rimorso : 335-372.

COCHIN Charles Nicolas, 1756, Observations upon the Antiquities of the Town of Herculaneum, discovered at the foot of Mount Vesuvius. London : Wilson \& Durham.

CORNELIO Tommaso, 1672, « An Extract of a Letter, written March 51672 by Dr. Thomas Cornelio, a Neapolitan Philosopher and Physician, to John Dodington Esquire, his Majesties Resident at Venice ; concerning some Observations made of persons pretending to be stung by Tarantula's : English'd cut of the Italian ", in Philosophical Transactions : Giving some Accompt of the Present Undertakings, Studies and Labours of the Ingenious in many Considerable Parts of the World, vol. III, London : Printed by T.R. for John Martyn at the Bell in St. Pauls Churchyard, Printer of the Royal Society : 4006-4007.

DE HEUSCH Luc, 1971, Pourquoi l'épouser ? et autres essais. Paris : Gallimard. 
DE MARTINO Ernesto, 1961, La terra del rimorso. Contributo a una storia religiosa del Sud. Milano : Il Saggiatore.

DEPREZ Stanislav, 1999, Il cervello relazionale. Neuroscienze e socio-antropologia. Caltanissetta-Roma : Salvatore Sciascia Editore

DE RAHO Francesco, 1908, Il tarantolismo : nella superstizione e nella scienza. Lecce : Tipografia Dante Alighieri.

EDELMAN Gérard, 1992, Bright Air, Brilliant Fire. On the Matter of Mind. New York : Basic Books.

GALLUCCIO Paul, 1752, Notizie intorno alla città sotterranea Ercolano e suoi monumenti antichi discoperti alle falde del monte Vesuvio. Roma : N. Palearini.

GORI Antonio Francesco, 1756, Admiranda antiquitatum Herculanensium descripta et illustrata, 2 vol. Roma : Tipografia Pallade.

JERVIS Giovanni, 1961, «Considerazioni neuropsichiatriche sul tarantismo », in de Martino, La terra del rimorso : 287-306

LANTERNARI Vittorio, 1960, Movimenti religiosi di libertà e di salvezza dei popoli oppressi. Milano : Feltrinelli.

LANTERNARI Vittorio, 1995, « Tarantismo », in Storia, antropologia e scienze del linguaggio X, 3, septembre-decembre : 67-92.

LANTERNARI Vittorio, 2000, «Tarantismo : vecchie teorie, saperi nuovi », in Di Mitri Gino Leonardo (dir.), Quarant'anni dopo de Martino. Il tarantismo, Atti del Convegno Internazionale di Studi (Galatina 24-25 octobre 1998), 2 vol. (I). Nardò : Besa Editrice : 119-134.

LAPASSADE Georges, 1976, Essai sur la transe. Paris : Éditions Universitaires Jean-Pierre Delarge. MACCHIORO Vittorio, 1924, Orfismo e paolinismo, Montevarchi : s.t.

MACCHIORO Vittorio, 1930, Zagreus. Studi intorno all'orfismo. Firenze : Vallecchi.

MAFFEI Scipione, 1748, Tre lettere del signor marchese Scipione Maffei la prima sopra il primo tomo di Dione [...]. La seconda sopra le nuove scoperte d'Ercolano. Verona : Stamperia del Seminario.

MARINONI Augusto, dir., 1952, Leonardo da Vinci. Scritti letterari. Milano : Rizzoli.

MECATTI Giuseppe Maria, [1752], Esame o sia confronto di ragioni addotte dall'autore delle Novelle Letterarie di Firenze dottor Giovanni Lami da Santacroce e dall'abate Giuseppe Maria Mecatti fiorentino sopra le pretese città di Pompei e d'Ercolano. Napoli : s.t.

MINA Gabriele, 2000, Il morso della differenza. Il dibattito sul tarantismo dal XIV al XVI secolo. Nardò : Besa Editrice.

MONTINARO Brizio, 1996, San Paolo dei serpenti. Analisi di una tradizione, Palermo : Sellerio Editore.

MORA George, 1963, « An Historical and Sociopsychiatric Appraisal of Tarantism and its Importance in the Tradition of Psychotherapy of Mental Disorders ", in Bulletin of the History of Medicine 37 : 417-439.

NOCERA Maurizio et Pierre FUMAROLA, dir., 1994, Tarantolismo. Roma : Sensibili alle foglie. PEPE Roberto, 2002, Tarante, veleni e guarigioni, Atti del Convegno Interdisciplinare (Lecce 31 ottobre 2000). Nardò : Ideemultimediali.

PRINCE Raymond, 1982, «Introduction » à Ethos.Journal of the Society for Psychological Anthropology $\mathrm{X}, 4: 299-302$. 
ROUGET Gilbert, 1990 [1980], La musique et la transe. Esquisse d'une théorie générale des relations de la musique et de la possession. Paris : Gallimard.

SPERBER Daniel, 1996, Explaining Culture. A Naturalistic Approach.Oxford : Blackwell.

VALLETTA Ludovico, 1706, De phalangio apulo. Opusculum in quo pleraque historice, pleraque Philosophice de hoc Insecto, ejusque miro veneno enarrantur, ac discutiuntur. Napoli : ex Tipographia De Bonis.

VENUTI Marcello, 1748, Descrizione delle prime scoperte dell'antica città d'Ercolano ritrovata vicino a Portici, villa della maestà del re delle Due Sicilie. Roma : Bernabo e Lazzarini.

VENUTI Marcello, 1749, Descrizione delle prime scoperte dell'antica città d'Ercolano. Venezia : L. Baseggio.

WHITEHOUSE Harvey, 1997, «Jungles and Computers : Neuronal Group Selection and the Epidemiology of Representations ». Journal of the Royal Anthropological Institute 2 : 99-116.

ZEMPLENI Andras, 1966, « La dimension thérapeutique du culte des rab, ndöp, tuuru et samp ; rites de possession chez les Lebou et les Wolof », in Psychopathologie africane a. II, 3 : 295-439.

\section{NOTES}

1. La varietas comportementale regroupe l'ensemble des comportements déterminés par l'action de la musique sur l'esprit, selon les doctrines philosophiques antiques qui établissent une complémentarité entre les harmonies et les remèdes destinés à guérir les dissonances psychiques. Pour une approche exhaustive de ce thème, voir Boccadoro (2002).

2. Le Bestiario vincien est daté d'environ 1494.

3. Il s'agit d'une anthologie des écrits concernants la tarentule par Guglielmo de Marra, Sante Ardoini, Leon Battista Alberti, Teseo Pini, Marsilio Ficino, Gaspare Visconti, Serafino Aquilano, Pietro Pomponazzi, Ferdinando Ponzetti, Pietro Andrea Mattioli, Girolamo Cardano, Giulio Cesare Scaligero, Giovan Battista della Porta, Girolamo Mercuriale, Tommaso Campanella,.

4. Littéralement « religion des origines anciennes "; ce terme latin renvoie de manière générique à l'ensemble de la spiritualité païenne antique et à ses croyances (pratiques cultuelles, cosmogonie, théogonie, etc.).

5. Le culte des corybantes était fondamentalement un culte de possession. Chaque esprit était associé à une couleur et à une musique déterminées, et il était invoqué dans les rituels par une mélodie et une danse spécifiques.

6. Il s'agit du processus de transpiration résultant de l'exercice prolongé de la danse effrénée.

7. L'aiôresis est le mouvement produit par le sujet assis sur une balançoire (image présente dans certains mythes grecs anciens); il est pratiqué par les tarentulés dans une perspective symbolique.

8. Il y a aussi une édition moderne de ce livre (Nocera et Fumarola 1994) comportant un article de G. Lapassade.

9. Le commentaire de Minasi se trouve aux pages 475-490 d'un traité - celui de Cataldantonio Atenisio Carducci - dont il n'est pas officiellement l'auteur (voir bibliographie).

10. Cf. p. 122-123: "St. Gervais in libro cui titulus est Mémoires historiques, refert apud Africanos, Tuneti scilicet, endemium esse Tarantismum spontaneum, seu a nullius insecti morsu dependentem, mulieribus potissimum familiarem, quo ad saltandum, et choreas agendas impelluntur, et huic morbo nomen est le Janon ; hos quidem motus tanquam convulsivos Auctor reputat; verum verosimile est, eos ejusdem generis, ac quos vere tarantati apud Apulos edere solent. » 
11. L'hyperoxie une hyperventilation produisant une augmentation excessive du taux d'oxygène dans le sang et, par conséquent, des états de conscience modifiés.

\section{RÉSUMÉS}

Dans son parcours sur la littérature biomédicale, sur les traités de spiritualité et sur les journaux du voyage scientifique du XVIII ${ }^{\mathrm{e}}$ siècle ayant pour objet le tarentisme de l'Italie méridionale, cette contribution tente de reconstruire le tissu de la perception et de la représentation de ce célèbre rituel de possession auprès des savants européens. Il en ressort un cadre anthropologique surprenant. Bien qu'il paraisse aujourd'hui incroyablement moderne dans ses modalités et dans les solutions des observations sur le terrain et parfois dans certaines intuitions interprétatives fulgurantes dans lesquelles apparaît l'attestation d'un phénomène de transe, ce modèle glissera peu de temps après dans la pénombre du renoncement herméneutique, livrant les possédés de la tarentule aux théories positivistes de l'aliénation. Les Illuministes ont, de toute façon, le mérite d'avoir récolté l'antique héritage mystérique du tarentisme et établi le principe de relations comparatives avec le monde afro-asiatique, qui devaient profitablement nourrir l'ethnomusicologie contemporaine et les autres sciences anthropologiques.

\section{INDEX}

Index géographique : Italie

\section{AUTEUR}

\section{GINO L. DI MITRI}

Gino L. Di Mitri a obtenu un doctorat en Histoire des sciences à l'Università degli Studi di Bari avec une thèse sur les changements du paradigme biomédical du tarentisme au XVIII ${ }^{\mathrm{e}}$ siècle, et un DEA en Histoire sociale et culturelle des savoirs et des pratiques de santé à l'Université de Genève avec un mémoire sur le grand-tour des médecins et des savants européens dans le Royaume de Naples. Ila publié bon nombre de monographies, d'essais et d'articles sur l'histoire de la transe et de la possession. Actuellement il collabore au Département d'études d'histoire de l'Università degli Studi di Lecce. 